\title{
Endothelial lipase: direct evidence for a role in HDL metabolism
}

\author{
Jonathan C. Cohen
}

McDermott Center for Human Growth and Development, Center for Human Nutrition, Department of Internal Medicine, University of Texas Southwestern Medical Center at Dallas, Dallas, Texas, USA

J. Clin. Invest. 111:318-321 (2003). doi:10.1172/JCI200317744.

For the past three decades, epidemiologic studies have consistently demonstrated an inverse relationship between plasma HDL cholesterol (HDL-C) concentrations and coronary heart disease (CHD) (1). Population-based studies have provided compelling evidence that low HDL-C levels are a risk factor for CHD (2-4), and several clinical interventions that increased plasma levels of HDL-C were associated with a reduction in CHD risk $(5,6)$. These findings have stimulated extensive investigation into the determinants of plasma HDL$C$ levels. Turnover studies using radiolabeled apolipoprotein A-I, the major protein component of HDL, suggest that plasma HDL-C concentrations are highly correlated with the rate of clearance of apolipoprotein AI (7). However, the metabolic mechanisms by which HDL are catabolized have not been fully defined. Previous studies in humans with genetic deficiency of cholesteryl ester transfer protein (8), and in mice lacking the scavenger receptor BI (SRBI) (9), have demonstrated that these proteins participate in the removal of cholesterol from HDL, while observations in individuals with mutations in hepatic lipase indicate that this enzyme hydrolyzes HDL triglycerides (10). In

\footnotetext{
Address correspondence to: Address correspondence to: Jonathan C. Cohen, Center for Human Nutrition, University of Texas Southwestern Medical Center at Dallas, 5323 Harry Hines Boulevard, Dallas, Texas 75390-9052, USA. Phone: (214) 648-4774; Fax: (214) 648-4837; E-mail:

jonathan.cohen@utsouthwestern.edu. Conflict of interest: The author has declared that no conflict of interest exists.

Nonstandard abbreviations used: HDL

cholesterol (HDL-C); coronary heart disease (CHD); scavenger receptor BI (SR-BI); endothelial lipase (LIPG).
}

this issue of the JCI, reports from laboratories of Tom Quertermous (11) and Dan Rader (12) now indicate that endothelial lipase (LIPG), a newly identified member of the lipase family, catalyzes the hydrolysis of HDL phospholipids and facilitates the clearance of HDL from the circulation.

Endothelial lipase was initially cloned by both of these laboratories using entirely different strategies. Quertermous and his colleagues identified endothelial lipase as a transcript that was upregulated in cultured human umbilical vein endothelial cells undergoing tube formation (13), whereas the Rader group cloned endothelial lipase as a transcript that was upregulated in the human macrophage-like cell line THP-1 exposed to oxidized LDL (14). Database searches revealed that endothelial lipase shows strong sequence similarity to lipoprotein lipase (44 percent identity) and hepatic lipase (41 percent identity), two well-characterized lipases that function at vascular endothelial surfaces. Critical motifs associated with lipase activity (GXSXG and the catalytic triad S169, D193, H274), and with heparin binding were strongly conserved $(13,14)$. Interestingly, in contrast to both lipoprotein lipase and hepatic lipase, endothelial lipase has little triglyceride hydrolase activity in vitro but instead cleaves fatty acids from the $s n-1$ position of phosphatidylcholine. In in vitro assays the enzyme is most active on lipids presented in HDL, although it will release fatty acids from all classes of lipoproteins (15). Consistent with this finding, adenovirus-mediated overexpression of endothelial lipase in LDL receptor-deficient mice reduced plasma concentra- tions of VLDL and LDL cholesterol by about 50 percent, whereas HDL-C decreased to almost zero in these animals (14). These data suggested that endothelial lipase may play a role in HDL catabolism.

To prove that endothelial lipase plays a physiological role in HDL metabolism, both groups assessed the effects of diminished endothelial lipase activity in vivo. The Quertermous group used gene targeting to inactivate $\mathrm{Lipg}$ in mice. $\mathrm{Lipg}^{-/}$mice were viable and fertile and had no obvious defects. Plasma cholesterol and phospholipid concentrations were increased by 69 percent and 52 percent respectively in male $\mathrm{Lipg}^{-1}$ mice. The changes in plasma cholesterol were due primarily to an increase in plasma HDL. Despite comparable increases in plasma phospholipid levels, the increase in plasma cholesterol levels in female $\mathrm{Lipg}^{-/}$mice was far more modest than that observed in male mice. To further evaluate the effect of varying endothelial lipase activity, Quertermous also generated mice expressing a human LIPG transgene. Consistent with the findings in endothelial lipase knockout mice, overexpression of $L I P G$ resulted in modest decreases in plasma phospholipid and cholesterol concentrations. Rader and colleagues inhibited endothelial lipase in mice by administering a polyclonal anti-murine endothelial lipase antibody, a strategy that has been used previously to assess the physiological roles of hepatic lipase and lipoprotein lipase. In wild-type mice, plasma cholesterol and phospholipid concentrations increased by about 25 percent within 24 hours of antibody administration. Inhibition of endothelial lipase activity elicited more pronounced changes in plasma lipid levels in hepatic lipase-deficient mice and in ApoAI transgenic mice. In the ApoAI transgenic mice, antibody administration was associated with a modest but statistically significant reduction in HDLphospholipid turnover.

These findings suggest that the metabolic pathways of the two major classes of lipoproteins, apolipoprotein B containing lipoproteins and HDL, may have more in common than was previously recognized, and that normal lipoprotein 


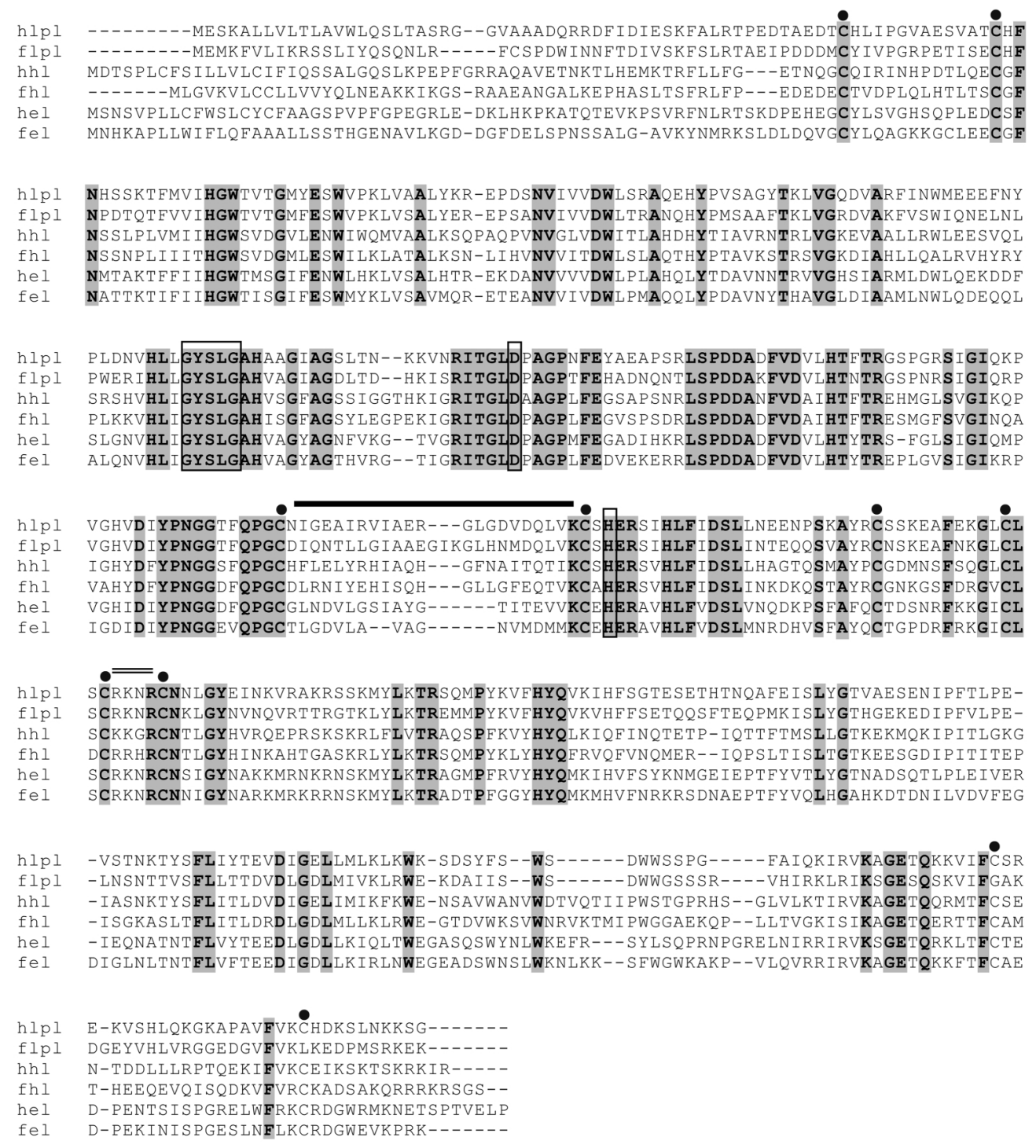

\section{Figure 1}

Sequence alignment of lipoprotein lipase ( $\mid \mathrm{pl})$, hepatic lipase ( $\mathrm{hl}$ ), and endothelial lipase (el) from human (h) and pufferfish Takifugu rubripes ( $f$ ). Amino acids that are identical in all three genes in both species are shaded in grey. The serine, aspartic acid, and histidine resides of the catalytic triad, as well as the lipase motif (GXSXG) are boxed. The conserved cysteine residues are denoted with closed circles. The lid domain is indicated with a single line, and the conserved heparin-binding region (BBXB) is indicated with a double line.

metabolism requires the action of three vascular endothelial lipases with distinct substrate specificities. The catabolic pathway for the apolipoprotein B containing lipoproteins includes two sequential steps; lipolysis and receptormediated endocytosis. Nascent triglyceride-rich lipoproteins secreted from the liver and intestine (chylomicrons and very low density lipoproteins) are delipidated first by lipoprotein lipase, which has a strong preference for triglycerides, and then by hepatic lipase which hydrolyzes both triglycerides and phospholipids. The triglyceride-depleted remnants of this delipidation cascade are removed from the circulation by receptor-mediated endocytosis. Consequently, patients lacking lipoprotein lipase accumulate very large, triglyceride-rich lipoproteins (16), whereas genetic defects in hepatic lipase lead to the accumulation of partially-delipidated lipoproteins of intermediate density (10). Now it appears that high density lipoprotein metabolism may also include both a lipolytic step, in which the principal lipid component (phosphatidylcholine) is hydrolyzed by endothelial lipase, and a receptor-mediated step, in which cholesterol is removed by selective uptake via the SR-BI.
The high degree of sequence conservation among the three vascular endothelial lipases suggests that they arose from a common ancestor and evolved distinct substrate specificities to accommodate the full spectrum of circulating lipoproteins. The duplication events that (presumably) gave rise to the three lipases must have occurred early in vertebrate evolution, since the Takifugu rubripes (pufferfish) genome encodes a putative ortholog for each lipase that is more similar to the corresponding human protein than to the other family members (Figure 1). Overall sequence identity among the 
six proteins is about 22 percent with the conserved amino acids clustered in motifs flanking the Ser-Asp-His residues of the catalytic triad. Eight of the ten cysteine residues thought to be involved in disulfide linkages are perfectly conserved, and a potential heparin-binding motif (BBXB, where $B$ is a basic residue and $X$ is any amino acid) is also strongly conserved in all six proteins. The lid region of the protein, which confers substrate specificity, shows little conservation between the different lipases but is highly conserved between the corresponding human and fugu enzymes. This finding suggests that three heparin-bound lipases with a common overall structure but distinct substrate specificities were already established in early vertebrates. Biochemical studies will be required to determine if these proteins have the same substrate preferences as their human orthologs.

The demonstration that endothelial lipase plays an important role in lipoprotein metabolism is an important milestone. However, several important aspects of endothelial lipase function remain to be addressed. Both of the current papers examined the effects of endothelial lipase deficiency on the concentration and composition of HDL. However the position of endothelial lipase action in the temporal sequence of events in HDL metabolism has not been determined. Does endothelial lipase act upstream of SR-BI, possibly priming HDL for interaction with the receptor? Or does the enzyme remove redundant phospholipids from HDL that have been depleted of cholesterol by SR-BI? The mechanism by which endothelial lipase reduces HDL levels is also not fully defined. Although the biochemical activity and location of the enzyme is consistent with a role in HDL catabolism, inhibition of the enzyme was associated with a very modest decrease in HDL phospholipid turnover, leading Rader and colleagues to speculate that endothelial lipase may limit the acquisition of phospholipids and cholesterol by HDL. Endothelial lipase may hydrolyze phospholipids on triglyceride-depleted remnant particles, preventing their transfer to HDL and thus limiting HDL production.

Perhaps the most important questions concerning endothelial lipase are: a) does it play an important role in HDL metabolism in humans, and b) does it play a significant role in atherogenesis. Given the known differences in HDL metabolism between mice and humans, observations in humans with genetically defined differences in endothelial lipase activity will be required to provide definitive answers. De Lemos et al. (17) have reported three rare alleles of endothelial lipase in subjects with high HDL-C concentrations that were not found in subjects with normal HDL levels. The sample sizes were too small to allow meaningful statistical analyses, and no evidence was provided that the variants segregated with HDL concentrations in families. Therefore further studies will therefore be required to determine if genetic variations that alter endothelial lipase activity are systematically associated with variation in plasma HDL concentrations and with atherosclerotic heart disease.

\section{Acknowledgments}

I thank Jay Horton for helpful discussions and Robert Barnes for assembling the fugu lipase orthologs. This work was supported by grants from the NIH (HL53917), the W.M. Keck Foundation, the LeDucq Foundation, and the D.W. Reynolds Cardiovascular Clinical Research Center.

1. 1993. National cholesterol education program. Second report of the expert panel on detection, evaluation and treatment of high blood cholesterol in adults. National Institutes of Health. Bethesda, Maryland, USA. IV:4-IV:5.

2. Miller, G.J., and Miller, N.E. 1975. Plasma-high-density-lipoprotein concentration and development of ischaemic heart-disease. Lancet. 1:16-19.

3. Gordon, T., Castelli, W.P., Hjortland, M.C., Kannel, W.B., and Dawber, T.R. 1977. High density lipoprotein as a protective factor against coronary heart disease. The Framingham Study. Am. J. Med. 62:707-714.

4. Wiklund, O., et al. 1980. Alpha-lipoprotein cholesterol concentration in relation to subsequent myocardial infarction in hypercholesterolemic men. Atherosclerosis. 37:47-53.

5. Frick, M.H., et al. 1987. Helsinki Heart Study: primary-prevention trial with gemfibrozil in middleaged men with dyslipidemia. Safety of treatment, changes in risk factors, and incidence of coronary heart disease. N. Engl. J. Med. 317:1237-1245.

6. Gordon, D.J., et al. 1989. High-density lipoprotein cholesterol and cardiovascular disease. Four prospective American studies. Circulation. 79:8-15.

7. Brinton, E.A., Eisenberg, S., and Breslow, J.L. 1994. Human HDL cholesterol levels are determined by apoA-I fractional catabolic rate, which correlates inversely with estimates of HDL particle size. Effects of gender, hepatic and lipoprotein lipases, triglyceride and insulin levels, and body fat distribution. Arterioscler. Thromb. 14:707-720.

8. Inazu, A., et al. 1990. Increased high-density lipoprotein levels caused by a common cholesteryl-ester transfer protein gene mutation. N. Engl. J. Med. 323:1234-1238.

9. Rigotti, A., et al. 1997. A targeted mutation in the murine gene encoding the high density lipoprotein (HDL) receptor scavenger receptor class B type I reveals its key role in HDL metabolism. Proc. Natl. Acad. Sci. USA. 94:12610-12615.

10. Hegele, R.A., et al. 1993. Hepatic lipase deficiency. Clinical, biochemical, and molecular genetic characteristics. Arterioscler. Thromb. 13:720-728.

11. Ishida, T., et al. 2003. Endothelial lipase is a major determinant of HDL level. J. Clin. Invest. 111:347-355. doi:10.1172/JCI200316306

12. Jin, W., Millar, J.S., Broedl, U., Glick, J.M., and Rader, D.J. 2003. Inhibition of endothelial lipase causes increased HDL cholesterol levels in vivo. J. Clin Invest. 111:357-362. doi:10.1172/JCI200316146.

13. Hirata, K., et al. 1999. Cloning of a unique lipase from endothelial cells extends the lipase gene family. J. Biol. Chem. 274:14170-14175.

14. Jaye, M., et al. 1999. A novel endothelial-derived lipase that modulates HDL metabolism. Nat. Genet. 21:424-428.

15. McCoy, M.G., et al. 2002. Characterization of the lipolytic activity of endothelial lipase. J. Lipid. Res. 43:921-929.

16. Santamarina-Fojo, S. 1998. The familial chylomicronemia syndrome. Endocrinol. Metab. Clin. North Am. 27:551-567.

17. deLemos, A.S., Wolfe, M.L., Long, C.J., Sivapackianathan, R., and Rader, D.J. 2002. Identification of genetic variants in endothelial lipase in persons with elevated high-density lipoprotein cholesterol. Circu lation. 106:1321-1326. 